\title{
Adult Rodent Neurogenic Regions: The Ventricular Subependyma Contains Neural Stem Cells, But the Dentate Gyrus Contains Restricted Progenitors
}

\author{
Raewyn M. Seaberg and Derek van der Kooy \\ Department of Anatomy and Cell Biology, University of Toronto, Toronto M5S 1A8, Canada
}

Neurogenesis persists in two adult brain regions: the ventricular subependyma and the subgranular cell layer in the hippocampal dentate gyrus (DG). Previous work in many laboratories has shown explicitly that multipotential, self-renewing stem cells in the subependyma are the source of newly generated migrating neurons that traverse the rostral migratory stream and incorporate into the olfactory bulb as interneurons. These stem cells have been specifically isolated from the subependyma, and their properties of self-renewal and multipotentiality have been demonstrated in vitro. In contrast, it is a widely held assumption that the "hippocampal" stem cells that can be isolated in vitro from adult hippocampus reside in the neurogenic subgranular layer and represent the source of new granule cell neurons, but this has never been tested directly. Primary cell isolates derived from the precise microdissection of adult rodent neurogenic regions were compared using two very different commonly used culture methods: a clonal colony-forming (neurosphere) assay and a monolayer culture system. Importantly, both of these culture methods generated the same conclusion: stem cells can be isolated from hippocampus-adjacent regions of subependyma, but the adult DG proper does not contain a population of resident neural stem cells. Indeed, although the lateral ventricle and other ventricular subependymal regions directly adjacent to the hippocampus contain neural stem cells that exhibit long-term self-renewal and multipotentiality, separate neuronal and glial progenitors with limited self-renewal capacity are present in the adult DG, suggesting that neuronspecific progenitors and not multipotential stem cells are the source of newly generated DG neurons throughout adulthood.

Key words: hippocampus; subgranular zone; subependyma; stem cells; progenitor cells; neurospheres
There are two regions in the adult rodent brain where neurogenesis occurs. Precursors from the subependyma surrounding the lateral ventricles proliferate and migrate along a rostral migratory stream to the olfactory bulbs, where they differentiate into granule cell interneurons (Lois and Alvarez-Buylla, 1993, 1994; Luskin, 1993). Second, precursors reside in the subgranular cell layer of the dentate gyrus (DG) in the hippocampus. These cells proliferate and migrate into the granule cell layer, where they differentiate into hippocampal granule cells (Altman and Das, 1965, 1966; Cameron et al., 1993; Kuhn et al., 1996; Palmer et al., 2000). We isolated the precursors from the subependyma and DG to test directly whether they exhibit similar properties in vitro and specifically to test whether they both exhibit stem cell characteristics. We define "stem cells" as single cells with long-term selfrenewal (self-renewal throughout the lifetime of the animal) and multipotentiality, "progenitors" as cells that exhibit less selfrenewal ability and unipotentiality or multipotentiality, and we use the term "precursor" to refer more generally to either a mixed or unknown proliferating population (Weiss et al., 1996).

The precursors that reside in the adult subependyma include a subpopulation of cells that exhibit the fundamental properties of neural stem cells: long-term self-renewal and multipotentiality

\footnotetext{
Received July 18, 2001; revised Nov. 29, 2001; accepted Dec. 18, 2001.

This work was supported by the Canadian Institutes of Health Research. We thank V. Tropepe, J. Ray, T. D. Palmer, and F. H. Gage as well as members of our lab for helpful comments on this manuscript. We also thank T. D. Palmer for helpful advice regarding AHP cell culture and preparation of AHP-conditioned media.

Correspondence should be addressed to Raewyn M. Seaberg, 8 Taddle Creek Road, Room 1105, Medical Sciences Building, University of Toronto, Toronto, Ontario M5S 1A8, Canada. E-mail: raewyn.seaberg@utoronto.ca.

Copyright (C) 2002 Society for Neuroscience $\quad 0270-6474 / 02 / 221784-10 \$ 15.00 / 0$
}

(Potten and Loeffler, 1990; Weiss et al., 1996). These subependymal cells display stem cell characteristics in vitro (Reynolds and Weiss, 1992; Morshead et al., 1994; Gritti et al., 1996; Tropepe et al., 1997; Chiasson et al., 1999), as well as self-renewal throughout the lifetime of the animal, even into senescence (Tropepe et al., 1997). In vivo, subependymal stem cells give rise to the progenitors that differentiate into olfactory bulb neurons (Lois and Alvarez-Buylla, 1993, 1994; Luskin, 1993).

Precursors exhibiting stem cell characteristics that reside in the adult hippocampus have also been isolated in vitro (Gage et al., 1995; Palmer et al., 1995, 1997). However, it has not been shown explicitly that these hippocampal stem cells reside in the subgranular cell layer of the DG, the site of adult neurogenesis. In vitro reports to date (Palmer et al., 1995, 1997) have studied cells derived from the dissociation of the entire hippocampus and thus have not separately investigated the properties of cells derived from the DG and those from neighboring subependymal neurogenic regions directly adjacent to the hippocampus. These regions include the third ventricle, posterior lateral ventricle, and the "hippocampal arch," which appears dorsally as a remnant of the caudal embryonic lateral ventricle that is not patent in the adult.

To test directly whether hippocampal stem cells reside in the DG, and whether they share properties with the subependymal stem cells, we specifically isolated precursors from these neurogenic regions in adult animals and compared their behavior using two different culture assays: a colony-forming (neurosphere) assay (Reynolds and Weiss, 1992; Chiasson et al., 1999) and a monolayer culture method (Gage et al., 1995; Palmer et al., 1995, 1997) that have been used for the isolation and study of subependymal and "hippocampal" stem cells, respectively. 


\section{MATERIALS AND METHODS}

Dissection and tissue preparation. Adult male CD1 mice (Charles River, St. Constant, Quebec, Canada), ages 6-8 weeks, and adult male Wistar rats (Charles River), ages 8-10 weeks, were used as indicated. Adult mice were killed by cervical dislocation; rats were deeply anesthetized with sodium pentobarbital and decapitated. The brains were removed, placed rostral-side up in $35 \mathrm{~mm}$ Petri dishes, and then covered with a $1.5 \%$ solution of low-gelling temperature agarose (Sigma, St. Louis, MO) at $38-40^{\circ} \mathrm{C}$ and then immediately placed at $4^{\circ} \mathrm{C}$ to harden the agarose solution. This supported the brain, permitting anatomy to remain intact and facilitating sectioning on a vibratome. Each brain was cut on a vibratome into viable $500 \mu \mathrm{m}$ coronal sections in ice-cold oxygenated artificial CSF (aCSF) that contained (in mM): $124 \mathrm{NaCl}, 5 \mathrm{KCl}, 1.3 \mathrm{MgCl}_{2}, 2 \mathrm{CaCl}_{2}, 26 \mathrm{NaHCO}_{3}$, and 10 D-glucose. Sections were placed into Petri dishes containing aCSF; rostral sections containing anterior lateral ventricle were kept separate from those containing dentate gyrus.

Under a Zeiss dissecting microscope, various brain regions from each section were precisely removed with fine surgical instruments. From the rostral sections, a $1.8 \times 0.2 \mathrm{~mm}$ strip of tissue containing both ependyma and subependyma was removed from the striatal (lateral) aspect of the anterior lateral ventricles. For all analyses, only subependymal spheres were counted, passaged, and differentiated (Chiasson et al., 1999). From more caudal sections containing hippocampus, the following regions were dissected: a $2.5 \times 0.2 \mathrm{~mm}$ strip of tissue just ventral to the corpus callosum, the hippocampal arch, a $1.3 \times 0.2 \mathrm{~mm}$ strip of tissue surrounding the posterior lateral ventricle, a $0.8 \times 1.2 \mathrm{~mm}$ piece of tissue surrounding the third ventricle, and a $1.3 \times 0.5 \mathrm{~mm}$ block of tissue containing both blades of the DG (and the entire subgranular cell layer) but excluding its most medial tip, which was included in the third ventricle dissection (see Fig. $1 A, B$ ). Dissected tissue from each region was placed into a test tube containing an enzyme solution as described previously (Chiasson et al., 1999) for 90 min at $37^{\circ} \mathrm{C}$ to facilitate dissociation of the cells. Tissues were then centrifuged at $1500 \mathrm{rpm}$ for $5 \mathrm{~min}$. The enzyme solution was removed, and serum-free media containing trypsin inhibitor $(15 \mathrm{mg} / \mathrm{ml}$ ovamucoid; Roche, Indianapolis, IN) was added. The tissues were mechanically triturated into a single-cell suspension with sterile fire-polished Pasteur pipettes and centrifuged again at $1500 \mathrm{rpm}$ for $5 \mathrm{~min}$. The ovamucoid solution was removed and replaced with chemically defined serum-free media (SFM) as described previously (Reynolds and Weiss, 1992; Tropepe et al., 1997).

For experiments involving early postnatal animals, pregnant CD1 mice and pregnant Wistar rats (Charles River) were obtained. The day of birth was counted as postnatal day (PND) 0, and all experiments were performed on PND1 or PND10. Newborn mice and rats were killed by decapitation, and the brains were removed under a dissecting microscope. The vibratome tissue preparation procedure for PND1 and PND10 animals followed that used for adults except that PBS was used instead of aCSF, and primary tissue was triturated in SFM into a single-cell suspension without previous enzyme treatment.

In vitro cell culture. The primary cell culture technique used for this study was the neurosphere assay (Reynolds and Weiss, 1992). Growth factors were used in SFM at final concentrations of $10 \mathrm{ng} / \mathrm{ml}$ fibroblast growth factor (FGF)2 (human recombinant; Sigma), $2 \mu \mathrm{g} / \mathrm{ml}$ heparin (Sigma), and $20 \mathrm{ng} / \mathrm{ml}$ epidermal growth factor (EGF) (mouse submaxillary; Sigma) unless noted otherwise. B27 supplement (Invitrogen, Burlington, $\mathrm{ON}, \mathrm{Canada}$ ) was added to primary tissue and passaged cultures unless noted otherwise. Cell viability was assessed using trypan blue exclusion $(0.4 \%$, Sigma), and was $>85 \%$, regardless of tissue region dissected or culture conditions used. Primary tissue was plated at 20 viable cells per microliter $(10,000$ cells per well) in uncoated 24 -well culture plates (Nunc, Naperville, IL). It has been demonstrated previously by mixing marked and unmarked cells that culturing cells at this density will result in clonal neurosphere colonies, as form in single-cell cultures, and that neurospheres do not arise as a result of cell aggregation at the cell culture densities used here (Morshead et al., 1998b; Tropepe et al., 2000). The total number of spheres that formed in each well was counted after $7 \mathrm{~d}$ in vitro (DIV) unless specified otherwise. New neurospheres (that were not present after 1 week) did not form after culturing tissue for longer time periods (up to 3 weeks) and repeatedly adding fresh media to the cultures (data not shown). The data are reported as the mean number of spheres plus the SEM formed per 10,000 cells or as the total number of spheres formed per brain dissection. To determine whether the spheres formed from each brain region were derived from stem cells, we assayed for self-renewal (single sphere passaging) as described previously (Tropepe et al., 1999). Self-renewal was also assayed (where indicated) by dissociating spheres in bulk and reculturing the cells at a constant density of 20 cells per microliter.

Control experiments were performed to exclude the possibility that the results obtained were a function of the neurosphere assay and not generalizable to other cell culture techniques. Thus, dissections were performed as described, and the tissue was plated in tissue culture flasks (Nunc) coated with polyornithine (Sigma) and laminin (Invitrogen) (Palmer et al., 1995, 1997) or in uncoated flasks (Gage et al., 1995). For the first $24 \mathrm{hr}$ the cells were cultured in SFM containing $10 \%$ fetal bovine serum (FBS) (Hyclone, Logan, UT), and the following day the media was replaced with SFM containing N2 supplement (Invitrogen) and $20 \mathrm{ng} / \mathrm{ml} \mathrm{FGF2}$. The procedure that we followed was identical to that described previously (Gage et al., 1995; Palmer et al., 1995, 1997). Control experiments with adult rat hippocampal-derived progenitor(AHP)-conditioned media were performed to exclude the possibility that inadequate glycosylated cystatin $\mathrm{C}(\mathrm{CCg})$ levels confounded our results; AHP-conditioned media was prepared as described (Taupin et al., 2000) and with the kind advice of T. D Palmer (Stanford University, Stanford, CA). Subsequently, the neurosphere assay was performed as described above, but with the absence of B27 supplement. Neurosphere assays were performed as well with AHPs (a gift from Dr. F. H. Gage and J. Ray, Salk Institute, Palo Alto, CA).

Immunocytochemistry. Multipotentiality was assayed by transferring single spheres to 24-well culture plates coated with MATRIGEL basement membrane matrix $(15.1 \mathrm{mg} / \mathrm{ml}$ stock solution diluted 1:50 in SFM; Becton Dickinson, Bedford, MA) in SFM containing 1\% FBS Various combinations of FBS, FGF2, B27, and other adhesive substrates (polyornithine and laminin) were tested, but $1 \%$ FBS on MATRIGEL was found to most reliably generate neurons and glia from subependymal neurospheres (data not shown). Wells were processed 7-8 d later using immunocytochemistry as described (Chiasson et al., 1999; Tropepe et al., 1999) for neurons and astrocytes. In contrast, for oligodendrocytes, the cultures were processed after $24 \mathrm{hr}$, and the cell permeabilization step was omitted. The following antibodies were used: anti-microtubule associated protein 2 (MAP2) mouse monoclonal (IgG) (1:1000; Roche), anti- $\beta$-tubulin isotype III mouse monoclonal (IgG) (1:500, Sigma), anti-glial fibrillary acidic protein (GFAP) rabbit polyclonal (IgG) (1:400; Chemicon, Temecula, CA), anti-O4 monoclonal (IgM) (1:100; Chemicon), FITC goat antirabbit (1:200; Jackson ImmunoResearch, West Grove, PA), rhodamine (TRITC)-conjugated goat anti-mouse (1:200; Jackson ImmunoResearch); anti-MAP2 mouse monoclonal (IgG1) (1:5000; RBI, Natick, MA), guinea pig anti-GFAP (1:500; Advanced ImmunoChemical, Long Beach, CA), FITC donkey anti-guinea pig (Chemicon), and Hoechst 33258 nuclear stain $(0.015 \mathrm{mg} / \mathrm{ml}$ stock solution diluted to $0.001 \mathrm{mg} / \mathrm{ml}$; Roche). Secondary-only wells were processed simultaneously using the identical protocol except that solutions did not contain primary antibodies. All secondary-only controls were negative for staining. The numbers of neurons and astrocytes generated per neurosphere were determined by counting the numbers of $\mathrm{MAP}^{+}$and $\mathrm{GFAP}^{+}$cells, respectively, as a percentage of Hoescht-positive nuclei in at least 14 random fields of differentiated cells. Fluorescence was visualized using a Nikon diaphot inverted microscope, and images were recorded with a Nikon COOLPIX digital camera.

$R T-P C R$. Total RNA was extracted from frozen differentiated sphere colonies (RNeasy extraction kit, Qiagen, Mississauga, Canada) and reverse transcribed. PCR for GFAP was performed with Taq DNA polymerase (MBI Fermentas, Burlington, Canada) in a GeneAmp PCR System 9700 (Applied Biosystems, Foster City, CA) using one step at $94^{\circ} \mathrm{C}, 2 \mathrm{~min}$; 35 cycles $\left(94^{\circ} \mathrm{C}, 1 \mathrm{~min} ; 55^{\circ} \mathrm{C}, 1 \mathrm{~min} ; 72^{\circ} \mathrm{C}, 1 \mathrm{~min}\right)$ and one extension step $\left(72^{\circ} \mathrm{C}, 10 \mathrm{~min}\right)$. PCR for neurofilament heavy-subunit (NF-H) was performed using one step at $95^{\circ} \mathrm{C}, 2 \mathrm{~min} ; 28$ cycles $\left(95^{\circ} \mathrm{C}, 0.5 \mathrm{~min} ; 56^{\circ} \mathrm{C}, 0.5\right.$ $\left.\min ; 72^{\circ} \mathrm{C}, 0.5 \mathrm{~min}\right)$ and one extension step $\left(72^{\circ} \mathrm{C}, 7 \mathrm{~min}\right)$. mRNA was treated with DNase (Qiagen) to exclude false results by DNA contamination. Forward and reverse primers used were $\left(5^{\prime}-3^{\prime}\right)$ : GTTGTGAAGGTCTATTCCTGGC and TCCCTTAGCTTGGAGAGCAA for GFAP (Bush et al., 1998) and AGCCTGAGGAGAAGCCCAAA and CGTAGCGTTCAGCATACATC for NF-H (Pernas-Alonso et al., 1996). The lengths of the amplified fragments were 150 and 452 bp for GFAP and NF-H, respectively. 
Figure 1. Dissection and rate of sphere formation of adult mouse neurogenic regions. $A, B$, Atlas images of adult mouse brain sections (Franklin and Paxinos, 1997) adapted to show the microdissection of viable $500 \mu \mathrm{m}$ vibratome sections used to isolate tissue from neurogenic regions. $A$, Coronal section through the anterior lateral ventricle $(a L V)$ with the dissected region highlighted. Note that this dissection includes both subependymal and ependymal tissue, but for the purposes of this study ependymal sphere formation was ignored. $B$, Coronal section through the hippocampus. Note that the dentate gyrus $(D G)$ dissection excludes all regions containing subependymal tissue; these regions were dissected and cultured separately. $3 r d V$, Third ventricle; $p L V$, posterior lateral ventricle; $H A$, hippocampal arch. This dissection scheme was used for both rats and mice.
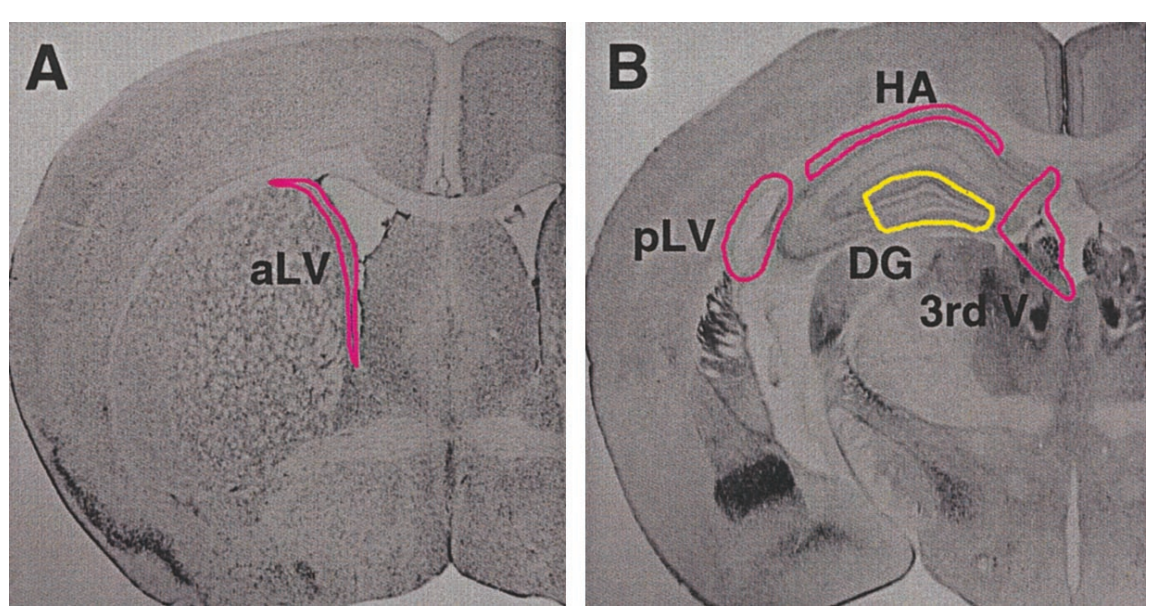

\section{RESULTS}

\section{Adult dentate gyrus cultures yield a very small number of sphere colonies}

Neurospheres can be generated from single neural stem cells that reside around the anterior lateral ventricle in the adult subependyma (Morshead et al., 1994; Chiasson et al., 1999). These neurospheres demonstrate the fundamental stem cell properties of self-renewal and multipotentiality; after dissociation of a single, clonally derived neurosphere, a few single cells proliferate to form new neurospheres (self-renewal) and when plated in differentiation conditions other cells from the single, clonally derived neurospheres differentiate to produce neurons and glia (multipotentiality). To determine whether the adult mouse DG contains stem cells that are similarly capable of generating neurospheres, we precisely isolated this region by microdissection of viable vibratome sections. Neurogenic brain regions containing subependymal cells are located directly adjacent to the hippocampus, and thus we dissected and separately cultured tissue from the posterior lateral ventricle, third ventricle, and hippocampal arch as part of our effort to avoid subependymal cell contamination of the DG culture (Fig. $1 A, B$ ). The hippocampal arch appears to be a remnant of the caudal embryonic lateral ventricle, which is not patent in the adult but contains a proliferating cell population that incorporates bromodeoxyuridine [as seen in Palmer et al. (2000) and our unpublished observations]. We have also dissected regions $\mathrm{CA} 1$ and $\mathrm{CA} 3$ of the hippocampus and the ventral pial surface for this assay and found that these regions never gave rise to spheres (data not shown).

The adult DG cultures did generate a very low number of spheres that were fewer in number (per 10,000 cultured cells, i.e., density of 20 cells per microliter) and significantly $(p<0.01)$ smaller in diameter $(135.11 \pm 7.53 \mu \mathrm{m})$ than those derived from the subependyma $(263.63 \pm 7.71 \mu \mathrm{m})$ of the anterior lateral ventricle, third ventricle, posterior lateral ventricle, or hippocampal arch. The average number of spheres generated from the DG per 10,000 cells was $0.54 \pm 0.1$ (Fig. $2 A$ ). This is a rate of sphere formation $\sim 120$-fold lower than that of anterior lateral ventricle subependymal cells.

\section{A similar small number of spheres are derived from dentate gyrus sections at each rostrocaudal level}

Usually, four to six coronal DG sections were obtained from a single animal, and the tissue fragments were pooled. To verify that the spheres did not arise from the accidental inclusion of a small amount of subependymal tissue in one of the sections, the
DG tissue retrieved from individual vibratome sections was cultured separately (rather than pooling tissue from multiple sections). We reasoned that if the spheres arising from DG cultures were a product of dissection error, they would more likely be derived from a single vibratome section that mistakenly included some subependymal cells rather than evenly distributed across rostrocaudal levels throughout the DG. In fact, the DG spheres were derived equally from rostral, middle, and caudal sections as revealed by one-factor ANOVA: $F_{(2,81)}=0.19 ; p>0.01$ (Fig. $2 B)$. This supports the idea that these colonies were truly formed by DG-derived cells and not by contaminating subependymal cells. Furthermore, as revealed in subsequent results described below, the DG and subependymal sphere colonies display different in vitro behavior, indicating that this small number of spheres was generated by DG and not subependymal cells.

It is important to emphasize that sphere formation is not in itself evidence of neural stem cell identity, as has been shown previously. For example, oligodendroglial progenitors have been shown to form free-floating "oligospheres," but these progenitors are not neural stem cells, because they exhibit limited selfrenewal ability and produce only oligodendrocytes (Zhang et al., 1998). Additionally, a subpopulation of cells derived only from the embryonic neural retina in the eye have the capacity to form spheres in vitro but are not capable of self-renewal and thus also do not represent neural stem cells (Tropepe et al., 2000).

In light of this, spheres were manipulated in vitro to assay for the stem cell properties of self-renewal and multipotentiality. In addition, a monolayer culture assay was used to ensure that results and subsequent conclusions were not specific to only one type of in vitro assay, as discussed below.

\section{Adult dentate gyrus-derived spheres do not demonstrate the stem cell property of self-renewal}

To determine whether the cells that had initiated the DG sphere colonies shared similar self-renewal characteristics with subependymal neural stem cells, primary spheres were individually dissociated into single cells and replated in identical media conditions. Through the use of this passaging assay, self-renewal ability is assessed by the formation of clonally derived secondary sphere colonies. The longevity of self-renewal ability can be investigated further by passaging single secondary spheres and assessing the formation of tertiary spheres, and so on. The number of spheres that form at each passage is an index of the number of times the primary sphere-initiating cell has symmetrically divided in vitro during primary sphere colony formation (Rey- 


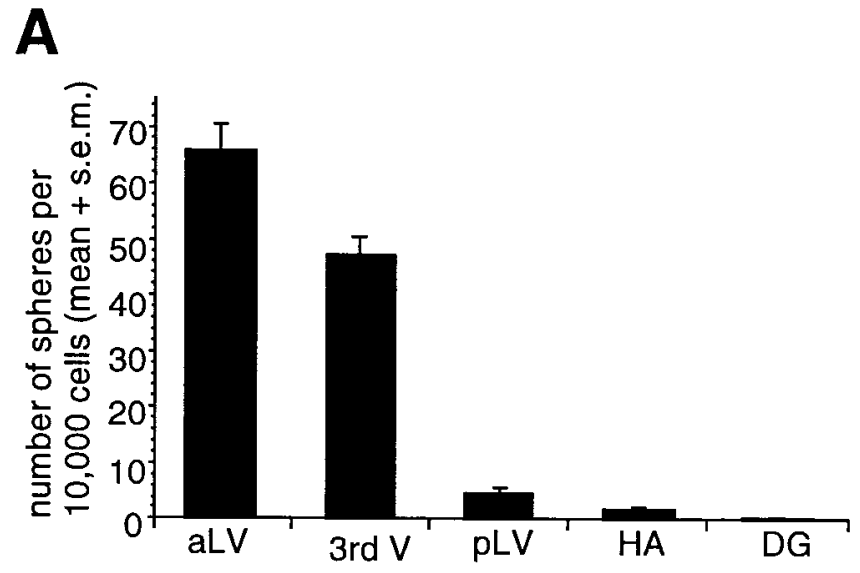

B

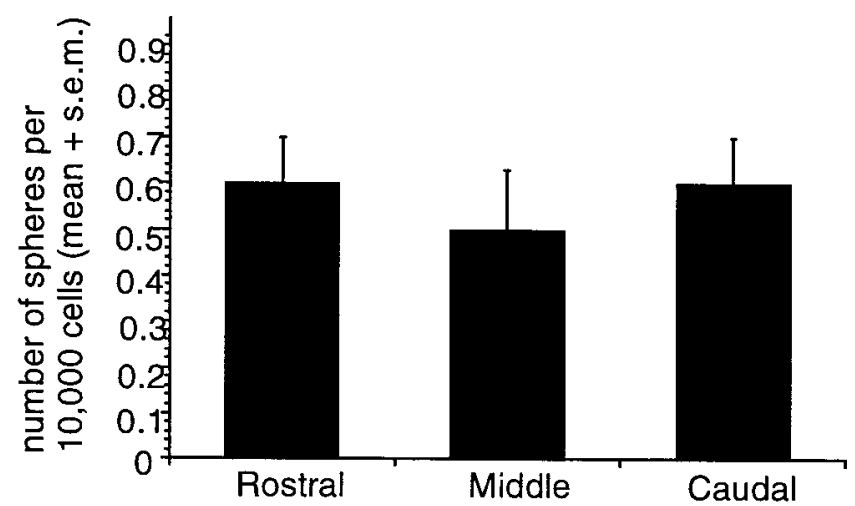

Figure 2. A, Comparison of the numbers of primary spheres generated from different neurogenic regions in the adult mouse brain. The data are expressed as the mean (+SEM) number of spheres generated per 10,000 cells plated (i.e., per well; density $=20$ cells per microliter). Tissue from each region ( $a L V$, anterior lateral ventricle; $p L V$, posterior lateral ventricle; $3 r d V$, third ventricle; $H V$, hippocampal arch; $D G$, dentate gyrus) was dissociated into single cells and plated in serum-free media containing EGF + FGF2 and B27 supplement; spheres were counted at 7 DIV. Note that the $a L V$ cells generate neurospheres at a rate $\sim 120$-fold higher than the $D G$ cells $(0.54 \pm 0.1)$, on the basis of data from $n>120$ animals and 10 separate experiments. $B$, Comparison of the number of spheres generated from adult dentate gyrus at different rostrocaudal levels. Rostral, middle, and caudal sections through the DG yield a similar number of sphere colonies. Tissue from each individual $500 \mu \mathrm{m}$ section $(n=85$ sections from $>20$ animals) through the DG was separately dissociated into single cells and cultured at 20 cells per microliter; spheres were counted at 7 DIV.

nolds and Weiss, 1996). Our results indicate that although virtually $100 \%$ of single, dissociated subependymal stem cell-derived anterior lateral ventricle neurospheres were capable of generating numerous new neurosphere colonies over multiple passages (Morshead et al., 1994; Tropepe et al., 1997; Chiasson et al., 1999), dissociated DG-derived spheres never gave rise to any secondary sphere colonies (Fig. $3 A, B$ ). Indeed, neurospheres derived from all dissected regions containing subependymal cells (posterior lateral ventricle, third ventricle, and hippocampal arch) were capable of giving rise to secondary neurospheres, demonstrating the self-renewal capability of the neurosphere-initiating cell from the subependyma. The only adult region studied from which nonpassagable spheres were generated was the DG; this
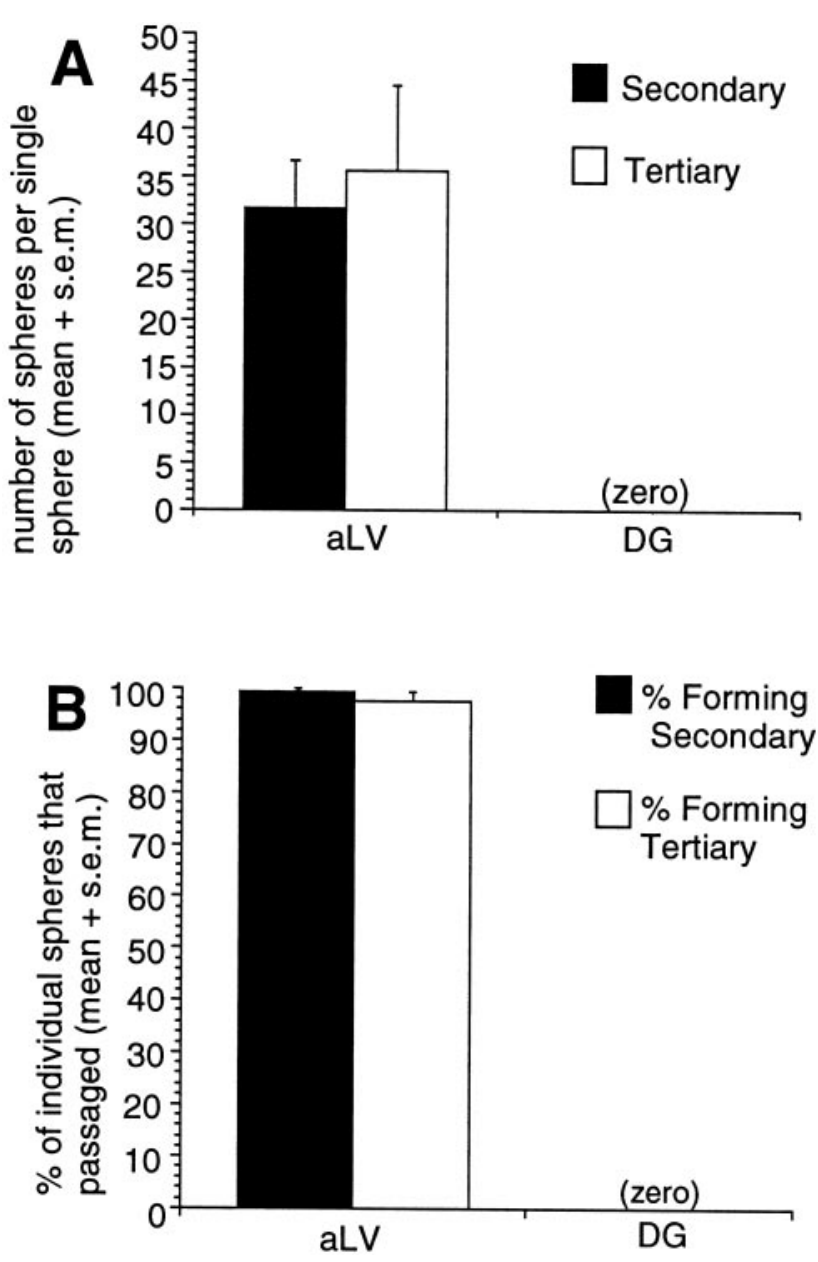

Figure 3. $A, B$, Comparison of the self-renewal ability of adult anterior lateral ventricle (subependymal) and dentate gyrus spheres. $A$, The data are expressed as the mean $(+\mathrm{SEM})$ number of spheres generated per single sphere dissociation. At each passage, individual spheres $(n>60$ spheres per condition) were dissociated per tissue culture well, and the number of sphere colonies that formed was counted after 7 DIV. Although the adult aLV neurosphere-initiating cells demonstrated selfrenewal by giving rise to secondary and tertiary neurospheres (as did the adult pLV, 3rd V, and HA neurospheres; data not shown), the adult DG sphere-initiating cells did not demonstrate self-renewal and did not give rise to secondary sphere colonies. Individual spheres were dissociated and replated in identical media conditions as were used for primary culture (EGF + FGF2 with B27 supplement). $B$, The data are expressed as the percentage of individual spheres that passaged to generate new spheres. Procedures were followed as described for $A$. Note that nearly $100 \%$ of adult aLV spheres give rise to new spheres at each passage.

strongly suggested that the cells from which DG spheres arise are not capable of self-renewal.

We considered the possibility that variability in cell density confounded the result that the relatively smaller DG spheres did not yield secondary colonies. A recent report (Taupin et al., 2000) has illustrated that at very low cell densities, factors elaborated by cultured cells are at insufficient concentrations for cell proliferation. Specifically, this report indicated that at cell densities of $<200$ cells per well (of a 96-well culture plate), cells required exogenous $\mathrm{CCg}$. To control for the possibility that such a factor was at an insufficient level in cultures of individually dissociated adult DG spheres, we pooled primary spheres derived from the anterior lateral ventricle and DG, dissociated them into single cells, and plated them at a constant density of 20 cells per 
microliter (4000 viable cells per well). Again, in contrast to the subependymal neural stem cell-derived colonies, the DG spheres did not give rise to any secondary sphere colonies. To more directly investigate whether insufficient $\mathrm{CCg}$ levels were responsible for the lack of self-renewing DG cells in our cultures, we prepared AHP-conditioned media (which does contain sufficient CCg for cell proliferation) (Taupin et al., 2000) and used it to supplement primary and secondary DG cell cultures. Even when cultured in AHP-conditioned medium, DG spheres did not give rise to secondary sphere colonies. This result further suggests that the DG sphere-initiating cells are not capable of long-term self-renewal and thus fail to meet one of the cardinal criteria for neural stem cells.

\section{The adult dentate gyrus contains separate neuronal and glial progenitors, but does not contain a population of neurosphere-initiating cells that demonstrate multipotentiality}

To determine whether the DG sphere-initiating cells (like subependymal neural stem cells) have the ability to generate multiple neural cell lineages, individual spheres from each neurogenic region were plated in differentiation conditions and then processed with immunocytochemistry to determine the relative percentages of neurons, astrocytes, and oligodendrocytes. Although $100 \%$ of individual adult anterior lateral ventricle neurospheres generated $\beta$ III-tubulin ${ }^{+}$neurons, $\mathrm{GFAP}^{+}$astrocytes, and $\mathrm{O}_{4}{ }^{+}$ oligodendrocytes, all of the adult DG spheres generated astrocytes but did not generate any neurons (i.e., did not express MAP2 or $\beta$ III-tubulin) or oligodendrocytes (Fig. 4, Table 1).

To further confirm the multipotentiality and unipotentiality of subependymal and DG sphere-initiating cells, respectively, we assayed differentiated sphere colonies for neuronal and glial gene expression using RT-PCR. We found that, in agreement with the aforementioned immunocytochemistry results, the subependymal sphere-initiating cell is multipotential because its differentiated progeny consist of both astrocytes (GFAP) and neurons (NF-H) (Fig. 4G). In contrast, the DG sphere-initiating cell is unipotential, because its differentiated progeny express GFAP but do not express NF-H.

The lack of neuron generation by adult DG spheres in vitro was unexpected, because it is well documented that there are cells in the adult DG that give rise to new neurons in vivo (Altman and Das, 1965, 1966; Cameron et al., 1993; Kuhn et al., 1996; Palmer et al., 2000). To determine whether neuronal precursors were present in the primary adult DG cultures, we isolated small (4-16 cells) clones from these $7 \mathrm{~d}$ cultures that had proliferated to a limited degree (in addition to the few DG spheres that formed, as described above). Small clones were distinguished from sphere colonies primarily by their low cell numbers, but also by morphology. Samples of these small clones were plated in differentiation conditions to determine which cell lineages could be derived from them. The small clones that survived immunocytochemical processing generated $\beta$ III-tubulin ${ }^{+}$neurons exclusively (Fig. $4 F$ ). Additional experiments were performed to determine whether isolates from other hippocampal regions intermediate between the subependyma and DG contained neuronal clones. Neuronal clones were not detected in isolates containing the hippocampal region $\mathrm{CA} 3$ or posterior lateral ventricle. However, a recent in vivo study detected a relatively small number of mitotically active neuronal cells in CA regions of the adult hippocampus (Rietze et al., 2000).

To assay for self-renewal, the small neuronal colonies were
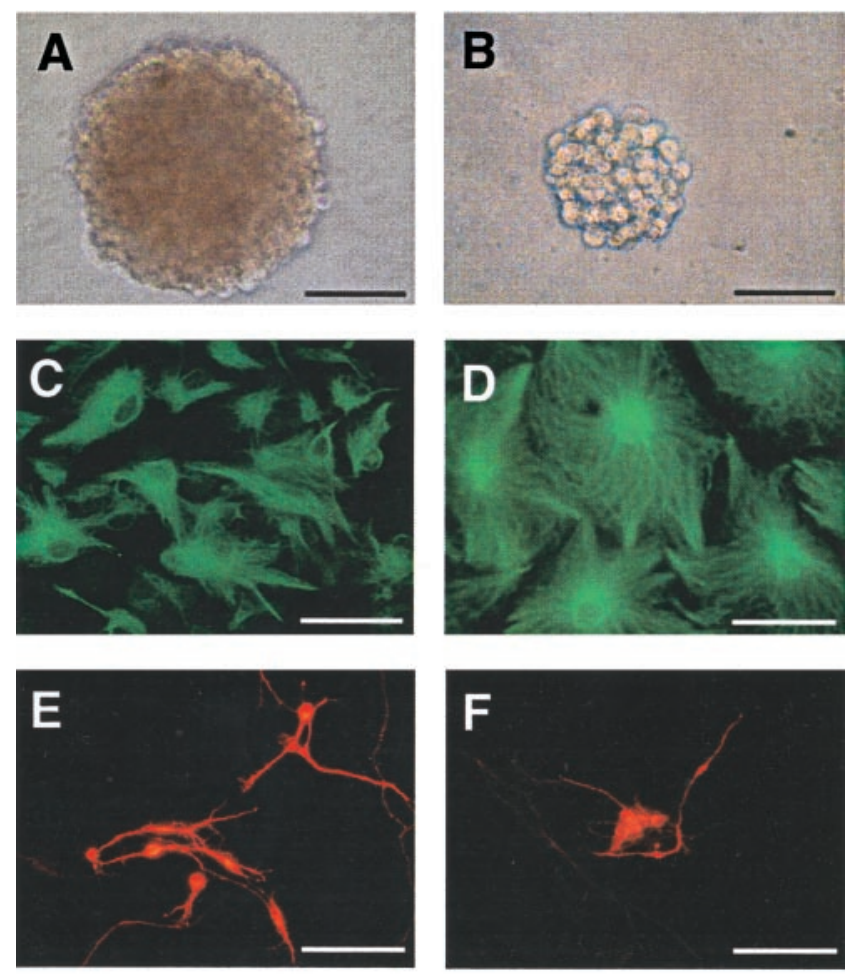

G

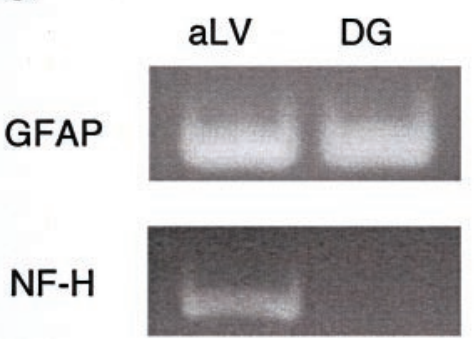

Figure 4. $A, B$, Comparison of the size of primary spheres derived from adult anterior lateral ventricle (subependyma) $(A)$ and dentate gyrus $(B)$. Note that the DG spheres are smaller than the aLV spheres. Scale bars, $100 \mu \mathrm{m}$. $C-G$, Comparison of the ability of adult aLV-and DG-derived primary spheres to contribute to different neural cell lineages. Primary adult aLV neurospheres generate both $\mathrm{GFAP}^{+}$astrocytes $(C)$ and $\beta \mathrm{III}-$ tubulin $^{+}$neurons $(E)$, whereas adult DG spheres generate only GFAP ${ }^{+}$ astrocytes $(D)$. Small adult DG clumps generate only $\beta$ III-tubulin ${ }^{+}$ neurons $(F)$. Note that although both cell types can be derived from adult aLV or DG cultures, the aLV-derived neurons and glia are generated by a common precursor, whereas the DG-derived neurons and glia are derived from separate progenitors. Individual spheres or clumps were plated on MATRIGEL basement membrane matrix in $1 \%$ FBS for $7-8$ DIV and then processed for immunocy tochemistry. Scale bars, $50 \mu \mathrm{m}$. $G$, Neuronal and glial gene expression were confirmed using RT-PCR. RNA was isolated from differentiated aLV and DG sphere colonies. Primers were used to detect GFAP (150 bp) and NF-H (452 bp). Sphere colonies derived from subependymal tissue $(a L V)$ generate both neuronal $(N F-H)$ and glial $(G F A P)$ progeny, whereas colonies derived from dentate gyrus cells $(D G)$ generate differentiated progeny that express GFAP but do not express NF-H. Data are representative of at least three separate experiments.

dissociated, but like the glia-producing DG spheres, they never produced secondary colonies. Thus, it was possible to isolate both neuronal and glial progenitors from adult DG tissue in vitro, but these two cell types were not generated from a common precursor cell. The adult DG does not contain neurosphere-initiating cells 


\begin{tabular}{|c|c|c|c|}
\hline \multicolumn{4}{|c|}{$\begin{array}{l}\text { Table 1. Mean percentages of neurons, astrocytes, and oligodendrocytes } \\
\text { generated from adult dentate gyrus and anterior lateral ventricle } \\
\text { neurospheres (mean } \% \pm \text { SEM) }\end{array}$} \\
\hline & $\begin{array}{l}\text { Neurons } \\
\left(\mathrm{MAP2}^{+}\right)\end{array}$ & $\begin{array}{l}\text { Astrocytes } \\
\left(\text { GFAP }^{+}\right)\end{array}$ & $\begin{array}{l}\text { Oligodendrocytes } \\
\left(\mathrm{O}^{+}\right)\end{array}$ \\
\hline $\mathrm{aLV}$ & $8.0 \pm 0.9$ & $88.7 \pm 1.4$ & $2.3 \pm 0.5$ \\
\hline DG & $0.0 \pm 0.0$ & $98.9 \pm 0.5$ & $0.0 \pm 0.0$ \\
\hline
\end{tabular}

Summary table illustrating the fundamental differences in cell lineage potential of spheres generated from different adult neurogenic brain regions. Spheres were cultured individually in MATRIGEL-coated wells in SFM and 1\% FBS. Numbers are expressed as a percentage of Hoescht-positive nuclei and are based on at least 14 random fields for each condition. aLV, Anterior lateral ventricle; DG, dentate gyrus.

that are capable of giving rise to multiple cell lineages, but it does contain separate populations of restricted progenitors.

\section{The absence of a self-renewing, multipotential cell population in the adult dentate gyrus is not an artifact of species or of cell culture technique}

Previous reports (Gage et al., 1995; Palmer et al., 1995, 1997) have suggested that the adult DG contains a population of selfrenewing, multipotential cells, often called AHPs (Suhonen et al., 1996). There are three main differences between the protocol in the referenced reports and that used in this study. The first is a difference in dissection: previous reports (Palmer et al., 1995, 1997) have used a gross dissection method that involved isolation of the entire hippocampus with no reported attempt to exclude surrounding subependymal tissues. Gage et al. (1995) reported an attempt to avoid the "subventricular zone," but we feel that this is difficult to accomplish reliably during gross hippocampal dissection. In contrast, our study has more precisely isolated the multiple neurogenic zones in the region of the hippocampus by microdissection of vibratome slices. The present dissection would appear to allow much better anatomical distinction of cell sources. Indeed, as a simple test of this in the present study, gross hippocampal dissection was performed, and multipotential, selfrenewing spheres were isolated that were not generated from more precise DG microdissection. This is an important result because it replicates the findings of Gage et al. (1995) and Palmer et al. (1995, 1997) using a different (neurosphere) culture assay: gross hippocampal dissection and culture lead to the isolation of a multipotential, self-renewing cell population. The key finding in the present study is that when the hippocampus is further divided into specific neurogenic regions, the neural stem cells are more precisely and correctly localized to regions adjacent to the hippocampus, rather than to the DG itself.

The second difference is that previous reports (Gage et al., 1995; Palmer et al., 1995, 1997) used rats, and the data presented here to this point have been derived from mice. The third major difference is in the use of monolayer cell culture techniques (Gage et al., 1995; Palmer et al., 1995, 1997) in previous reports, and the use of nonadherent neurosphere culture conditions in the present study.

We have addressed the species difference by performing identical experiments in rats as well as mice, and the results remain unchanged. Adult rat DG yielded a very small number of spheres (1.5 \pm 0.3 spheres per 10,000 viable cells) in comparison to regions containing subependyma, such as the anterior lateral ventricle (199 \pm 13 spheres per 10,000 viable cells). Furthermore, these sphere colonies did not passage to generate secondary spheres, and in differentiation conditions they produced $\mathrm{GFAP}^{+}$ astrocytes but did not produce neurons. In contrast, adult rat subependymal cells from the anterior lateral ventricle (and posterior lateral ventricle, third ventricle, and hippocampal arch) gave rise to neurospheres that were multipotential and capable of generating secondary neurospheres (data not shown). Therefore, we can conclude that the difference between previous results and the results of the present study is not attributable to a species difference; indeed, our adult rat data extend our initial findings from adult mice.

To address the potential confounding effect of different (monolayer vs neurosphere) cell culture techniques, we applied the identical culture techniques and media conditions as used in aforementioned reports [techniques followed according to both Gage et al. (1995) and Palmer et al. (1995, 1997); see Materials and Methods] to adult tissue isolated by our dissection methods. It seemed possible that the use of serum, higher growth factor concentration, different media supplement, or perhaps an adherent substrate permitted the survival of a multipotent, selfrenewing cell population from the DG that did not survive the serum-free, nonadherent culture conditions of the sphere assay. After following the referenced monolayer culture method (testing both coated and uncoated tissue culture plates), the adult subependymal cell-containing cultures became confluent, whereas the adult DG cell cultures, even when maintained for an extended period of time, never became $>40-50 \%$ confluent, perhaps suggesting a lower proliferative or self-renewal potential of the DG cells. After 3-4 weeks of monolayer culture, the surviving cells were transferred to our standard neurosphere assay conditions. These adult mouse anterior lateral ventricle subependymal cell cultures generated multipotential neurospheres, whereas the adult mouse DG cell cultures did not. Importantly, the same result was derived from both of these different culture protocols (Table 2). Interestingly, the AHPs derived from monolayer cultures of entire hippocampi (Gage et al., 1995; Palmer et al., 1995, 1997; Suhonen et al., 1996) readily formed many sphere colonies in vitro, as discussed below.

\section{A clonal cell line of adult hippocampal precursors displays different in vitro characteristics compared with primary adult rat dentate gyrus cultures}

Our data strongly suggest that the adult mouse DG does not contain a population of neurosphere-initiating cells with neural stem cell characteristics. However, as discussed above, other studies have reported the generation of self-renewing, multipotential AHPs derived from adult rats and the maintenance of these progenitors as clonal cell lines (Gage et al., 1995; Suhonen et al., 1996; Palmer et al., 1997). To help understand these fundamental differences between our primary adult rat cell cultures and monolayer-cultured AHPs, we compared them directly by plating the cells in a constant density neurosphere assay in both the presence and absence of growth factors. In contrast to the prevalent (although unpublished) notion that hippocampal cells do not readily form neurospheres, in our hands, AHPs generated a large number of spheres even in the absence of growth factors $(789 \pm 57)$. Furthermore, the AHPs produced higher numbers of spheres than primary rat tissue, as might be expected of a cell line enriched for neural progenitors (Fig. 5). Most interesting was the comparison between primary adult rat DG and anterior lateral ventricle and AHPs. In the absence of growth factors, the primary adult DG did not produce any spheres. However, the primary adult anterior lateral ventricle-derived subependymal cells and AHPs both generated sphere colonies in the absence of growth factors. These data suggest that the behavior of AHPs more 
Table 2. Summary of dissection technique and culture conditions used for the comparison of adult subependymal and dentate gyrus primary cell isolates

SE dissection

DG dissection

Gross HC dissection

$\mathrm{EGF}+\mathrm{FGF} 2+\mathrm{H}+\mathrm{B} 27$ (uncoated dishes)

$10 \%$ FBS; FGF2 + N2 (uncoated dishes; or

polyornithine/laminin coated)

$10 \%$ FBS; FGF2 + N2 + AHP-conditioned media

(uncoated dishes; or polyornithine/laminin coated)

Summary table illustrating the various dissection techniques and culture conditions used for the comparison of primary adult subependymal and dentate gyrus cells. A simplified version of the results is also illustrated where "+" represents the isolation of a multipotent, self-renewing cell in the tissue cultures from the various experimental conditions. Importantly, cell viability in all culture conditions was high. SE, Subependyma; DG, dentate gyrus; HC, hippocampus; EGF, epidermal growth factor; FGF2, fibroblast growth factor 2; H, heparin; B27, B27 supplement; N2, N2 supplement; FBS, fetal bovine serum; AHP, adult hippocampal progenitor.

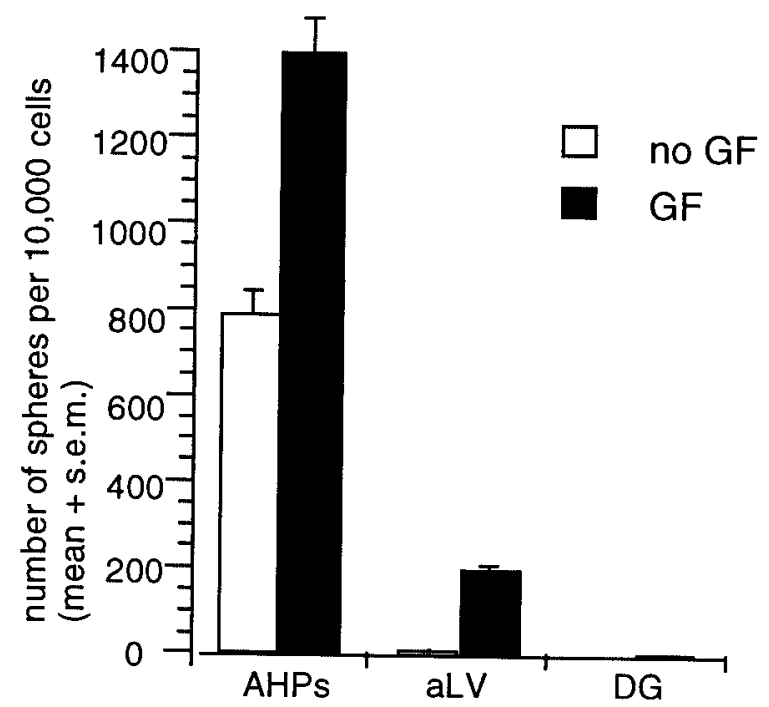

Figure 5. Comparison of the sphere-forming ability of primary tissue from adult rat neurogenic regions and a clonal cell line of adult hippocampal progenitors in the presence and absence of growth factors $(G F)$. The data are expressed as the mean $(+\mathrm{SEM})$ number of spheres generated per 10,000 cells plated (i.e., per well; density $=20$ cells per microliter). Cells were plated in either serum-free media $(n o G F)$ or serum-free media with EGF and FGF2 $(G F)$. AHPs were compared with primary adult rat tissue ( $D G$, dentate gyrus; $a L V$, anterior lateral ventricle). Resultant spheres were counted at 7 DIV. Note that both AHPs and primary aLV spheres $(12 \pm 2)$ were generated in $n o G F$, whereas zero spheres arose from adult DG in the absence of growth factors $(0.0 \pm 0.0)$. A small number of DG spheres were generated in $G F(1.5 \pm 0.3)$.

closely resembles that of primary adult rat subependymal cells than primary adult rat DG cells and that the previous reports of adult hippocampal stem cells in fact may have assayed cells derived from adjacent subependymal tissue and not from the DG itself.

\section{The early postnatal (PND1) dentate gyrus contains a population of neurosphere-initiating cells that demonstrate multipotentiality; this population declines with postnatal age}

Because the sphere-initiating cell is relatively rare in the adult DG, we reasoned that it might be possible to isolate a larger number of progenitor cells by dissecting out the DG during a stage of development when a large number of new cells are being born. Most ( $85 \%)$ of the DG granule cell neurons are generated postnatally, but by the end of the first 2 postnatal weeks the rate of granule cell birth has diminished (Schlessinger et al., 1975). In light of these findings, we decided to isolate the DG (and the

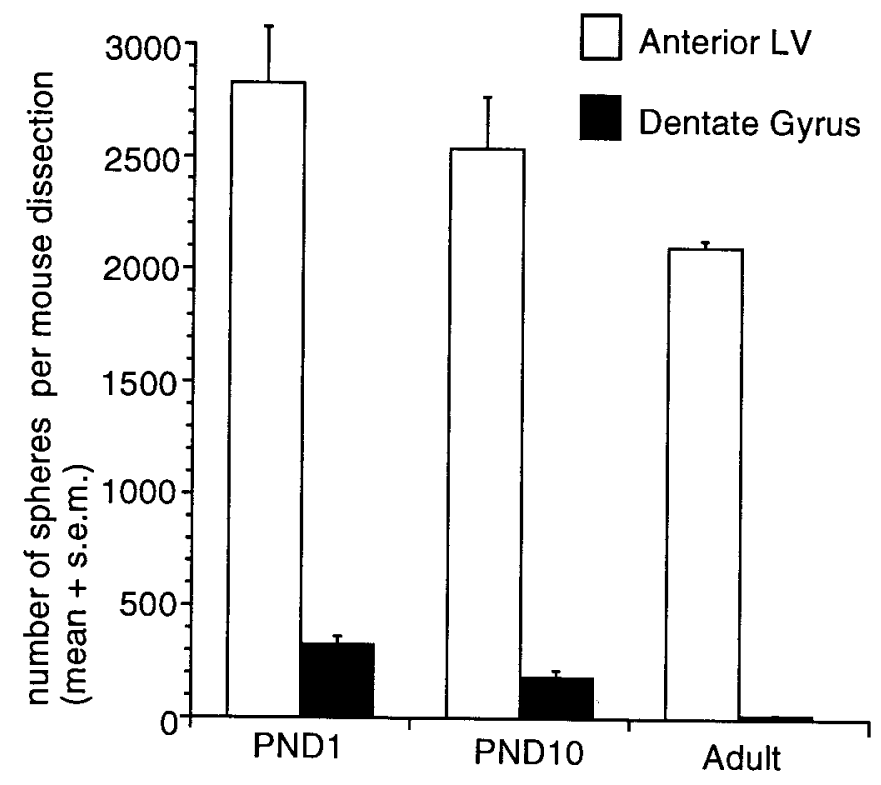

Figure 6. Comparison of the numbers of spheres formed from PND1, PND10, and adult anterior lateral ventricle (subependyma) and dentate gyrus. The data are expressed as the mean (+SEM) number of spheres generated per brain dissection. Tissue was dissected from adult, PND1, and PND10 brains ( $n>40$ animals from 5 separate experiments) from viable vibratome sections and cultured in EGF + FGF2 with B27 supplement at 20 cells per microliter. Spheres were counted at 7 DIV. Cells from the PND1 DG generated 30-fold more spheres than adult DG cells, whereas cells from the PND1, PND10, and adult aLV did not generate significantly different numbers of spheres.

subependyma-containing regions: anterior lateral ventricle, third ventricle, and posterior lateral ventricle) from early postnatal (PND1) mice in a manner identical to that described for the adult experiments. Indeed, the PND1 DG generated 30-fold more spheres than the adult DG. To control for differences in the numbers of postmitotic cells present in the neurogenic regions at different ages, sphere numbers were compared in terms of the total number of spheres generated per entire dissected region from each individual animal (rather than the average number per 10,000 viable cells) (Fig. 6). These DG spheres were similar in size $(p>0.01)$ to those derived from PND1 anterior lateral ventricle $(212.0 \pm 9.4$ and $216.4 \pm 8.4 \mu \mathrm{m}$, respectively). Moreover, spheres from both regions generated neurons, astrocytes, and oligodendrocytes when placed in differentiation conditions (data not shown). This result provided a positive control for the adult DG data and, interestingly, suggested that although the adult DG does not contain multipotential progenitors, a subpopu- 
lation of such cells may be present transiently in the early postnatal DG. Similarly, it has recently been demonstrated that oligodendrocyte progenitors from early postnatal optic nerve cultures can generate multipotential spheres after extensive growth factor manipulation in vitro (Kondo and Raff, 2000). Oligodendrocyte progenitors from the optic nerve thus may also represent a transient progenitor population that can be isolated from the early postnatal animal but is not maintained into adulthood (unpublished observations).

To test whether the number of multipotential sphere-initiating cells that can be isolated from the immature DG changes over the first 2 postnatal weeks, we isolated and assayed the DG of PND10 animals. The number of spheres that can be isolated from the PND10 DG was intermediate between the PND1 and adult numbers (Fig. 6). The PND1 and PND10 anterior lateral ventricle also generated more sphere colonies than adult anterior lateral ventricle, but the difference between the numbers of anterior lateral ventricle neurospheres generated at these three ages was not statistically significant, as revealed by one-factor ANOVA: $F_{(2,76)}=0.99 ; p>0.01$. In contrast to the anterior lateral ventricle neurospheres, the numbers of spheres isolated from the DG show a progressive decrease from PND1 to PND10 to adult $\left(F_{(2,65)}=\right.$ 13.03; $p<0.01)$. Thus, the large decline in the number of multipotential sphere-initiating cells as a function of postnatal age is specific to the DG. Furthermore, no remnant of this multipotential population persists in the DG into adulthood; this stands in direct contrast to the self-renewing, multipotential stem cell population that can be isolated from the subependyma throughout postnatal life and does not undergo diminution with age, even in senescent animals (Tropepe et al., 1997).

\section{DISCUSSION}

The results of this study demonstrate that the adult DG contains a discrete subpopulation of cells that can form sphere colonies when isolated in vitro. However, these sphere-initiating cells do not self-renew in culture and generate only glial progeny; this subpopulation of cells does not exhibit the fundamental stem cell properties of long-term self-renewal and multipotentiality. We also have isolated adult DG neuronal progenitors that similarly show very limited self-renewal ability and are restricted to the neuronal lineage. Moreover, we have replicated these results with a monolayer culture technique. This result appears to be in direct contrast to previous studies that reported the isolation of hippocampal neural stem cells (Palmer et al., 1995, 1997) and implies that the new neurons generated in vivo in the adult DG (Altman and Das, 1965, 1966; Cameron et al., 1993; Kuhn et al., 1996; Palmer et al., 2000) arise from a population of restricted neuronal progenitors rather than from a population of stem cells.

In an attempt to explain these conflicting results, we identified and addressed a number of potential confounding factors. These include effects of cell density when assessing self-renewal and differences between the protocol used in the present study and that used in previous studies (Gage et al., 1995; Palmer et al., 1995, 1997). A series of experiments that controlled for potential confounding effects of cell density and $\mathrm{CCg}$ level (Taupin et al., 2000) when assaying for self-renewal did not alter the aforementioned result that the adult DG progenitors do not exhibit selfrenewal in vitro and thus do not meet one of the key neural stem cell criteria.

Other differences between previous reports of hippocampal stem cell isolation (Gage et al., 1995; Palmer et al., 1995, 1997) and the present study include the use of rats (previously) and of mice (in the present study) and differences in dissection and cell culture technique. We addressed the species issue by performing identical experiments and replicating our results in rats. Indeed, this series of experiments provides further confirmation and extension of our results in mice and suggests that our findings may be generalized to a wide range of species.

Differences in dissection and cell culture technique were addressed by comparing both gross hippocampal dissections and monolayer culture techniques (Gage et al., 1995; Palmer et al., $1995,1997)$ with our microdissection procedure and use of nonadherent neurosphere culture conditions. Again, these experiments replicated our initial results: specific DG isolates do not contain self-renewing, multipotential sphere-initiating cells, whereas gross hippocampal dissections (that included ventricular subependyma) do contain such cells. Furthermore, these selfrenewing, multipotential cells can be detected in gross hippocampal isolates whether the tissue is exposed to monolayer or suspension culture techniques (Table 2).

Regardless of the source of stem cells isolated in previous reports, there are other alternative explanations for the present results that have not been ruled out here. These include the possibility that even at 20 cells per microliter, our culture conditions lack a factor (other than $\mathrm{CCg}$ ) necessary for the survival or proliferation of adult DG cells that is not required for the survival and proliferation of adult subependymal cells. It is possible that stem cells do exist in the adult DG, but our culture conditions are simply not appropriate for their isolation or proliferation. This is an interesting possibility, because such contrasting behavior to the subependymal neural stem cells would suggest that there are region-specific stem cells in different areas of the adult brain. However, this same neurosphere assay has been sufficient for the isolation of two other very different types of neural stem cells: those residing in the adult subependyma (Chiasson et al., 1999) and the retinal stem cells of the adult ciliary margin (Tropepe et al., 2000). It is possible that the putative adult DG neural stem cell could be an exceptional case, but there is currently no evidence to support this possibility.

Because we can isolate very small colonies of neuron-producing progenitors from adult DG specific cultures, we propose a model whereby the in vivo adult neurogenesis (and gliogenesis) occurring in the DG results from the limited proliferation and differentiation of pools of restricted neuronal (and restricted glial) progenitors. Furthermore, we suggest that large numbers of progenitors migrate into the developing DG perinatally, each capable of only a limited number of divisions. These separate progenitors divide sequentially in vivo to generate new neurons (or new astrocytes) in the DG into adulthood. Because this pool of progenitors decreases over time, DG neurogenesis declines with age. These unipotential adult progenitor cells may be derived from the multipotential progenitors that can be isolated at PND1, or they may be generated perinatally directly from ventricular zone stem cells without passing through a multipotential stage.

In support of this model, there is evidence from in vivo studies that the cells responsible for adult DG neurogenesis exhibit properties more characteristic of restricted progenitors than of neural stem cells. It is known from studies of progenitor and stem cell populations in the subependyma that an age-related decrease occurs in the number of restricted progenitors but not in the number of neural stem cells (Tropepe et al., 1997), thus indicating that progenitor and stem cell numbers are differentially regulated with age. Interestingly, an age-related decrease has been found in the proliferation of cells in the adult DG (Seki and Arai, 1995; 
Kuhn et al., 1996), suggesting that this cell population is more likely to consist of restricted progenitors than of neural stem cells (although the remaining progenitors can be induced to proliferate after adrenalectomy in older animals) (Cameron and McKay, 1999).

Our model is supported by the age-related decrease in the number of multipotent, self-renewing sphere colonies that can be isolated from the DG (results from the present study). This number declines postnatally until in the adult DG multipotential sphere colonies can no longer be isolated, and only separate unipotent neuronal and glial progenitor populations with very limited self-renewal ability remain. Importantly, the number of multipotent, self-renewing sphere colonies isolated from the subependyma surrounding the anterior lateral ventricle does not change significantly with age. Into adulthood, and even into senescence (Tropepe et al., 1997), a constant population of subependymal neurospheres that exhibit the stem cell properties of long-term self-renewal and multipotentiality can be isolated in vitro.

There are a number of alternatives to our proposed model. It is possible that the PND1 DG spheres were initiated by stem cells, but by adulthood these stem cells have migrated out of the DG proper (and thus would not be included in the adult DG dissection) and have become confined to the surrounding subependyma. Although there is no evidence for this regressive model of postnatal DG stem cell migration, it remains possible in theory. It is possible also that the surrounding subependymal stem cells are the source of DG neuronal progenitors in vivo. This seems unlikely, because none of the studies that have examined ventricular cell migration with retroviral labeling assays (Luskin, 1993; Morshead et al., 1994, 1998a; Doetsch and Alvarez-Buylla, 1996) have reported migration of a retrovirally labeled cell into the DG. A final alternative mentioned previously is that the early postnatal DG progenitors are in fact neural stem cells that persist into adulthood, but by this stage they have changed their characteristics to the extent that they can no longer be isolated by our surgical and culture techniques.

Recent studies have provided evidence that neural stem cells in the adult subependyma may be GFAP $^{+}$(Doetsch et al., 1999; Laywell et al., 2000). The results of the present study neither support nor oppose these reports, because we did not prospectively assay the GFAP immunoreactivity of sphere-forming cells. Certainly, the isolation of a multipotential neurosphere-forming cell from the adult subependyma is in agreement with the results of the present study. However, another recent publication (Seri et al., 2001) suggests that a subpopulation of $\mathrm{GFAP}^{+}$cells in the adult hippocampus generate neurons. Nevertheless, none of the experiments in this report were done clonally, and thus there is presently no in vivo evidence that individual $\mathrm{GFAP}^{+}$cells in the subgranular layer are capable of generating both differentiated neurons and differentiated astrocytes within this hippocampal region. Indeed, our data suggest that separate cells in the adult subgranular layer generate neurons and astrocytes. Moreover, there is no evidence presented by Seri et al. (2001) that the hippocampal $\mathrm{GFAP}^{+}$cell that they follow in vivo possesses the second stem cell characteristic of long-term self-renewal. Interestingly, in accordance with our finding of unipotential glial and neuronal progenitors in the adult DG cell isolates, unipotential neuronal progenitors also have been isolated in vitro from adult human DG (Roy et al., 2000).

The present results may have substantive implications for adult neural stem cell research. We suggest that rather than two sepa- rate (subependymal and subgranular layer) stem cell populations, there is in fact only one population of authentic (subependymal) neural stem cells in the adult brain, although the subependymal stem cells and subgranular layer progenitors have a common anatomical origin during development. This is important because the adult in vivo stem cell niche may be restricted to a single subependymal tissue type, perhaps aiding in the identification and characterization of neural stem cells and their stem cell niche.

\section{REFERENCES}

Altman J, Das GD (1965) Autoradiographic and histological evidence of postnatal hippocampal neurogenesis in rats. J Comp Neurol 124:319-336.

Altman J, Das GD (1966) Autoradiographic and histological studies of postnatal neurogenesis. I. A longitudinal investigation of the kinetics, migration and transformation of cells incorporating tritiated thymidine in neonate rats, with special reference to postnatal neurogenesis in some brain regions. J Comp Neurol 126:337-389.

Bush TG, Savidge TC, Freeman TC, Cox HJ, Campbell EA, Mucke L, Johnson MH, Sofroniew MV (1998) Fulminant jejuno-ileitis following ablation of enteric glia in adult transgenic mice. Cell 93:189-201.

Cameron HA, McKay RDG (1999) Restoring production of hippocampal neurons in old age. Nat Neurosci 2:894-897.

Cameron HA, Woolley CS, McEwen BS, Gould E (1993) Differentiation of newly born neurons and glia in the dentate gyrus of the adult rat. Neuroscience 56:337-344.

Chiasson BJ, Tropepe, V, Morshead CM, van der Kooy D (1999) Adult mammalian forebrain ependymal and subependymal cells demonstrate proliferative potential, but only subependymal cells have neural stem cell characteristics. J Neurosci 19:4462-4471.

Doetsch F, Alvarez-Buylla A (1996) Network of tangential pathways for neuronal migration in adult mammalian brain. Proc Natl Acad Sci USA 93:14895-14900.

Doetsch F, Caille I, Lim DA, Garcia-Verdugo JM, Alvarez-Buylla A (1999) Subventricular zone astrocytes are neural stem cells in the adult mammalian brain. Cell 97:703-716.

Franklin KBJ, Paxinos G (1997) The mouse brain in stereotaxic coordinates. San Diego: Academic.

Gage FH, Coates PW, Palmer TD, Kuhn HG, Fisher LJ, Suhonen JO, Peterson DA, Suhr ST, Ray J (1995) Survival and differentiation of adult neuronal progenitor cells transplanted to the adult brain. Proc Natl Acad Sci USA 92:11879-11883.

Gritti, A, Parati EA, Frolichsthal, P, Galli, R, Wanke, E, Faravelli, L, Morassutti DJ, Roisen, F, Nickel DD, Vescovi AL (1996) Multipotential stem cells from the adult mouse brain proliferate and self-renew in response to basic fibroblast growth factor. J Neurosci 16:1091-1100.

Kondo T, Raff M (2000) Oligodendrocyte precursor cells reprogrammed to become multipotential CNS stem cells. Science 289:1754-1757.

Kuhn HG, Dickinson-Anson H, Gage FH (1996) Neurogenesis in the dentate gyrus of the adult rat: age-related decrease of neuronal progenitor proliferation. J Neurosci 16:2027-2033.

Laywell ED, Rakic P, Kukekov VG, Holland EC, Steindler DA (2000) Identification of a multipotential astrocytic stem cell in the immature and adult mouse brain. Proc Natl Acad Sci USA 97:13883-13888.

Lois C, Alvarez-Buylla A (1993) Proliferating subventricular zone cells in the adult mammalian forebrain can differentiate into neurons and glia. Proc Natl Acad Sci USA 90:2074-2077.

Lois C, Alvarez-Buylla A (1994) Long-distance neuronal migration in the adult mammalian brain. Science 264:1145-1148.

Luskin MB (1993) Restricted proliferation and migration of postnatally generated neurons derived from the forebrain subventricular zone. Neuron 11:173-189.

Morshead CM, Reynolds BA, Craig CG, McBurney MW, Staines WA, Morassutti D, Weiss S, van der Kooy D (1994) Neural stem cells in the adult mammalian forebrain: a relatively quiescent subpopulation of subependymal cells. Neuron 13:1071-1082.

Morshead CM, Craig CG, van der Kooy D (1998a) In vivo clonal analyses reveal the properties of endogenous neural stem cell proliferation in the adult mammalian forebrain. Development 125:2251-2261.

Morshead CM, Tropepe V, Hadjantonakis AK, Nagy A, van der Kooy D (1998b) The non-cell autonomous sphere forming ability of neural stem cells. Soc Neurosci Abstr 24:1528.

Palmer TD, Ray J, Gage FH (1995) FGF-2-responsive neuronal progenitors reside in proliferative and quiescent regions of the adult rodent brain. Mol Cell Neurosci 6:474-486.

Palmer TD, Takahashi J, Gage FH (1997) The adult rat hippocampus contains primordial neural stem cells. Mol Cell Neurosci 8:389-404.

Palmer TD, Willhoite AR, Gage FH (2000) Vascular niche for adult hippocampal neurogenesis. J Comp Neurol 425:479-494.

Pernas-Alonso R, Schaffner AE, Perrone-Capano C, Orlando A, Morelli 
F, Hansen CT, Barker JL, Esposito B, Cacucci F, di Porzio U (1996) Early upregulation of neurofilament gene expression in developing spinal cord of the wobbler mouse mutant. Mol Brain Res 38:267-275.

Potten CS, Loeffler M (1990) Stem cells: attributes, cycles, spirals, pitfalls and uncertainties. Lessons for and from the crypt. Development 110:1001-1020

Reynolds BA, Weiss S (1992) Generation of neurons and astrocytes from isolated cells of the adult mammalian central nervous system. Science 255:1707-1710.

Reynolds BA, Weiss S (1996) Clonal and population analyses demonstrate that an EGF-responsive mammalian embryonic $\mathrm{CNS}$ precursor is a stem cell. Dev Biol 175:1-13.

Rietze R, Poulin P, Weiss S (2000) Mitotically active cells that generate neurons and astrocytes are present in multiple regions of the adult mouse hippocampus. J Comp Neurol 424:397-408.

Roy NS, Wang S, Jiang L, Kang J, Benraiss A, Harrison-Restelli C, Fraser R AR, Couldwell WT, Kawaguchi A, Okano H, Nedergaard M, Goldman S (2000) In vitro neurogenesis by progenitor cells isolated from the adult human hippocampus. Nat Med 6:271-277.

Schlessinger AR, Cowan WM, Gottlieb DI (1975) An autoradiographic study of the time of origin and the pattern of granule cell migration in the dentate gyrus of the rat. J Comp Neurol 159:149-176.

Seki T, Arai Y (1995) Age-related production of new granule cells in the adult dentate gyrus. NeuroReport 6:2479-2482.

Seri B, Garcia-Verdugo JM, McEwen BS, Alvarez-Buylla A (2001) As- trocytes give rise to new neurons in the adult mammalian hippocampus. J Neurosci 21:7153-7160.

Suhonen JO, Peterson DA, Ray J, Gage FH (1996) Differentiation of adult hippocampus-derived progenitors into olfactory neurons in vivo. Nature 383:624-627.

Taupin P, Ray J, Fischer WH, Suhr ST, Hakansson K, Grubb A, Gage FH (2000) FGF-2-responsive neural stem cell proliferation requires CCg, a novel autocrine/paracrine cofactor. Neuron 28:385-397.

Tropepe V, Craig CG, Morshead CM, van der Kooy D (1997) Transforming growth factor- $\alpha$ null and senescent mice show decreased neural progenitor cell proliferation in the forebrain subependyma. J Neurosci 17:7850-7859.

Tropepe V, Sibilia M, Ciruna BG, Rossant J, Wagner EF, van der Kooy D (1999) Distinct neural stem cells proliferate in response to EGF and FGF2 in the developing mouse telencephalon. Dev Biol 208:166-188.

Tropepe V, Coles BLK, Chiasson BJ, Horsford DJ, Elia AJ, McInnes RR, van der Kooy D (2000) Retinal stem cells in the adult mammalian eye. Science 287:2032-2036.

Weiss S, Reynolds BA, Vescovi AL, Morshead C, Craig CG, van der Kooy D (1996) Is there a neural stem cell in the mammalian forebrain? Trends Neurosci 19:387-393.

Zhang S-C, Lipsitz D, Duncan ID (1998) Self-renewing canine oligodendroglial progenitor expanded as oligospheres. J Neurosci Res 54: 181-190. 